\title{
Avaliação da Função Renal: Creatinina e outros Biomarcadores ${ }^{\star}$
}

\section{Evaluation of Renal Function: Creatinine and other Biomarkers}

\author{
Márcia Cristina da Silva Magro ${ }^{1}$, Maria de Fátima F. Vattimo ${ }^{2}$
}

RESUMO

JUSTIFICATIVA E OBJETIVOS: Os biomarcadores são ferramentas diagnósticas discriminatórias entre o estado de saúde e doença. O objetivo deste estudo foi reconhecer a aplicação clínica dos biomarcadores de função renal na prática clínica, com a finalidade de disponibilizar conhecimento do avanço diagnóstico da lesão renal aguda (LRA).

MÉTODO: Estudo descritivo de levantamento bibliográfico de periódicos indexados de 1975 a outubro de 2006, por meio das bases de dados LILACS e PubMed.

RESULTADOS: Foram disponibilizados 505 artigos nas bases de dados PubMed e 6 nas do LILACS. Foram selecionados 106 artigos e, após leitura na íntegra, apenas 69 traduziam a abordagem temática pretendida.

CONCLUSÕES: Nesse levantamento foi verificado que apesar do progresso na compreensão dos mecanismos celular e molecular envolvendo a LRA, ainda existe um hiato entre a compreensão e a aplicação de tratamentos efetivos e específicos na prevenção e controle dessa síndrome.

Unitermos: biomarcador biológico, insuficiência renal aguda

1. MS, Enfermeira da Unidade de Recuperação Cardíaca do Instituto do Coração do HC-FMUSP e Doutora da Escola de Enfermagem da USP

2. MS, PhD, Professora da Escola de Enfermagem da USP

*Recebido do Instituto do Coração do Hospital de Clínicas da Faculdade de Medicina da Universidade de São Paulo (HC-FMUSP), São Paulo, SP

Apresentado em 23 de janeiro de 2007

Aceito para publicação em 10 de maio de 2007

Endereço para correspondência:

Márcia Cristina da Silva Magro

Alameda Santos, 663/83, BL B Cerqueira César

01419-001 São Paulo, SP

Fone: (11) 3284-1376

E-mail: ppmmagro@uol.com.br

(C)Associação de Medicina Intensiva Brasileira, 2007

\section{SUMMARY}

BACKGROUND AND OBJECTIVES: Biomarkers are diagnostic tools which discriminate between the good health and the illness. This study had as objective to recognize the clinical application of the renal function biomarkers in the clinical practice, in order to inform the diagnostic advances of the acute kidney injury (AKI).

METHODS: Descriptive study of bibliographical survey of indexed periodicals from 1975 to October, 2006, by means of the LILACS and PubMed databases.

RESULTS: Were available 505 articles from the bibliographical survey in the PubMed database and 6 in the Lilacs database. 106 articles were selected and, after full reading, only 69 referred to the intended thematic approach.

CONCLUSIONS: In this survey, it was verified that despite the progresses in the molecular and cellular mechanisms understanding related to $\mathrm{AKI}$, there is still a gap between the comprehension and the application of effective and specific therapeutics in the prevention and control of this syndrome.

Key Words: acute renal failure, biological biomarker

\section{INTRODUÇÃO}

A lesão renal aguda (LRA) é freqüentemente observada em pacientes internados e sua prevalência está aumentando, principalmente na população idosa, com múltiplas comorbidades e em pacientes com doenças consideradas graves como neoplasia entre outras ${ }^{1}$. Ela representa uma condição clínica causada pela diminuição ou perda da capacidade de manutenção dos equilíbrios ácido-base e hidroeletrolítico.

Apesar do progresso terapêutico e tecnológico observado nos últimos anos, o prognóstico da LRA permanece sombrio. Ela é considerada uma complicação freqüente nas unidades de terapia intensiva (UTI) e seu diagnóstico tardio repercutem em elevada mortalidade.

Sabidamente, as manifestações clínicas da LRA são 
incipientes, silenciosas e se confundem com sinais clínicos de diversas outras morbidades, sendo freqüentemente percebidas quando constatadas alterações em exames laboratoriais de rotina, como a uréia e principalmente a creatinina.

Contudo, elevações nos níveis séricos da creatinina são atualmente os sinais mais indicativos de comprometimento da função renal. Apesar de representar a principal estratégia de identificação dessa síndrome, a creatinina é considerada um teste específico, entretanto tardio, pouco sensível e impreciso. Ela se altera apenas quando já existe perda de aproximadamente $50 \%$ da função renal. A contraposição ao uso convencional desses indicadores se apercebe de situações mais desfavoráveis como é o caso na disfunção como a insuficiência hepática, na qual não há indicadores diretos de função e o seu diagnóstico e a conseqüente intervenção, tardam mais ainda. Em nenhum dos dois casos deve haver, contudo, resignação científica nem tampouco clínica.

O relativo descompasso da creatinina com o real estado funcional e sua baixa sensibilidade e especificidade se traduzem em diagnóstico tardio e tratamento mais tardio ainda, medidas de prevenção próxima de zero e, por fim, alta mortalidade. Tal panorama tem deixado marcas precisas na epidemiologia da LRA.

Esse cenário desfavorável da LRA tem gerado então questionamentos sobre conceitos até então intocáveis. Um deles é a busca para identificação de biomarcadores mais precisos da função renal. Há de se conhecer indicadores de função renal, interpretados por toda equipe profissional, que tenham desempenho discriminatório superior àqueles mencionados. Já se sabe acerca desses novos testes.

Genericamente, biomarcadores são ferramentas que podem fornecer alguma informação necessária, especialmente quando usado em conjunto com dados clínicos e laboratoriais ${ }^{2,3}$. Um biomarcador deve consistir em indicador de processos biológicos, patogênicos ou resposta farmacológica para um tratamento terapêutico ${ }^{3}$.

A busca por novos biomarcadores para detecção da lesão renal está se desenvolvendo rapidamente com o avanço da tecnologia. Com melhores biomarcadores, possivelmente, tornará viável a detecção precoce da lesão renal, a identificação de lesões subclínicas, o fornecimento de informação prognóstica do curso da doença, a identificação dos segmentos mais afetados, a avaliação da resposta para determinados tratamentos e a classificação dos pacientes de risco para lesão renal ${ }^{4}$.
O objetivo deste estudo foi apresentar o resultado de um levantamento bibliográfico sobre os novos biomarcadores de função renal para que se possa reconhecer a sua aplicação na prática clínica, no sentido de disponibilizar conhecimento do avanço no diagnóstico da lesão renal aguda, que poderá refletir-se na tomada de decisão precoce junto ao paciente em risco para LRA.

\section{MÉTODO}

Este estudo é de caráter descritivo, baseado em levantamento bibliográfico de periódicos indexados.

Foi realizada busca bibliográfica utilizando as bases de dados LILACS (Literatura Técnica-Científica em Ciências da Saúde) e PubMed, envolvendo os periódicos do período de 1975 até outubro de 2006, referentes à literatura existente sobre os biomarcadores de função renal, utilizando como descritores (DeCS e MeSH): indicadores biológicos, insuficiência renal aguda, sendo as linguagens portuguesa e inglesa empregadas de acordo com a exigência das bases de dados

\section{RESULTADOS}

A análise do material bibliográfico obtido através da busca nas bases de dados permitiu verificar que a produção científica com pesquisas envolvendo biomarcadores de função renal é extensa, porém, não consensual.

Foram encontrados 505 artigos referentes ao tema. Da PubMed selecionou-se 100 e após a análise individual e leitura na íntegra dos estudos, apenas 68 artigos abrangiam o tema biomarcadores de lesão renal. Enquanto do LiLacs apenas um artigo foi utilizado. Foram incluídos todos os estudos de abordagens experimental e clínica.

Através desse levantamento foi identificado um perfil conciso de 29 biomarcadores de lesão renal, estudados em pesquisas com modelos animais e estudos clínicos nos últimos anos, dentre eles destacaram-se a Interleucina 18 (IL-18), a interleucina-6, a interleucina-8, o hidrato de carbono (HNK-1), o Malondialdeido urinário (MDA), a Cistatina C, o Dendrimer-enhanced MRI, a Glutathione alpha-transferase e pi-transferase, o Neutrophil gelatinase-associated Lipocalin (N-GAL), a Protein P53, o Proatrial natriuretic peptide (1-98), a Urinary actin, a $N$-acetyl-beta- $D$-glucosaminidase, a alpha-1-microglobulin, o Kidney Injury molecule1 (KIM-1), a Retinol Binding Protein (RBP), a Neutral endopeptidase (NEP), a Beta 2-microglobulin, urinary, o Sodium/hydrogen exchanger isoform 3 (NHE3), o 
Neutrophil CD11b, Spermidine/Spermine N(1)-acetyltransferase (SSAT), a Cysteine rich protein 61 (CYR61, CCN1), o 1,5-anhydroglicitol, a Adenosina deaminase binding protein, a Glycosyl transferase e entre as enzimas de lesão tubular encontraram-se a Gamma-Glutamyl transpeptidase (Gama-GTP), a Alanine aminopeptidase (AAP), a alkaline phosphatase (AP), a Leucine aminopeptidase e dipeptidyl peptidase IV (DPP).

$\mathrm{Na}$ classificação dos biomarcadores, alguns são destinados preferencialmente à estimativa da taxa de filtração glomerular, como a cistatina $\mathrm{C}$, outros refletem a função renal (KIM-1, NHE3 e actina), enquanto outros mostram inflamação associada com a lesão renal (Interleucinas 6,8 e 18) ${ }^{5,6}$. Há ainda o grupo de proteínas urinárias como a Retinol binding protein (RBP), a $N$-acetyl Glucosaminidase e a1-microglobulina, como indicadores mais sensíveis de disfunção ou lesão tubular 7,8 .

Dentre os biomarcadores descritos, observou algumas tendências de ênfase, iniciando-se pela Neutrophil gelatinase-associated lipocalin (NGAL). Trata-se de uma enzima presente na urina e no sangue. Observou-se que em crianças, a lesão renal aguda pode ser detectada em até duas horas após cirurgia cardíaca, por aumento substancial dessa enzima no sangue ou na urina. Pode ser ainda também detectada na presença de lesão renal em período que antecede a manifestação clássica pela creatinina ${ }^{9}$. Entretanto, ainda são necessários estudos multicêntricos prospectivos em populações maiores, de diferentes idades para validar esses resultados ${ }^{10}$. Novos estudos que avaliem fatores de interferência em seus níveis urinários ou plasmáticos serão providentes ${ }^{11}$.

Vale ressaltar que a elevada excreção de proteínas e enzimas de baixo peso molecular (proteínas tubulares) tem sinalizado situações de lesão tubular proximal. Nessa função, destacam-se a $\alpha 1$ e $\beta 1$-microglobulina, a Cistatina C, a Retinol Binding Protein (RBP) ${ }^{12,13}$, a $\alpha$ glutathione S-transferase (GST), a $\gamma$-glutamiltransferase (GGT), o Lactato desidrogenase (LD) e a $N$-acetyl- $\beta-D$ glucosaminidase (NAG) ${ }^{12,14,15}$.

A neutral endopeptidase (NEP) é livremente filtrada pelo glomérulo, sendo 99,9\% reabsorvida pelo túbulo proxi$\mathrm{mal}^{8}$. Representa um tipo de enzima tubular localizada na membrana endotelial do túbulo renal proximal e é excretada na urina. Sendo então, indicador de lesão tubular e a RBP, um indicador de função tubular. Enquanto, o $\mathrm{NHE}^{3}$ é a forma de permutador $\mathrm{Na}+/ \mathrm{H}+$ mais abundante na membrana apical, responsável pela reabsorção de grande parte do $\mathrm{NaCl}$ e do $\mathrm{NaHCO}^{3}$ filtrado nos glomérulos. Tem papel essencial na homeostase de volume, ácido-base e na determinação dos níveis da pressão arterial $^{16}$.

A IL-18 urinária pode servir como indicador de lesão tubular proximal em ATN. Ela é uma citocina pró-inflamatória liberada em resposta a lesão tubular ${ }^{17}$. A aplicação clínica desse teste pode ser substancial, já que exige técnicas confiável, precisa e rápida e ser prontamente mensurável utilizando-se kits de imunoensaio (Elisa).

Já a cistatina $C$ é um inibidor da cisteíno proteinase, produzida por células nucleadas em taxa constante. Ela é livremente filtrada pelo glomérulo, reabsorvida e metabolizada, mas não secretada pelos túbulos ${ }^{18,19}$. Ela pode detectar a LRA um ou dois dias antes da creatinina e, além disso, não é afetada por baixas doses de hormônio tireoidiano e deficiência ou excesso de corticóide ${ }^{5}$. A técnica utilizada para sua dosagem tem sido a nefelometria, no sangue ou na urina.

A Kidney Injury Molecule-1 (KIM-1) é uma proteína transmembrana que está presente no túbulo renal proximal e é superegulada e excretada na urina na vigência de uma lesão. Em indivíduos normais e sem a lesão renal ela não está presente. A elevação dessa enzima sugere o início de um processo patológico em células do epitélio tubular proximal, sendo assim considerada um sensível marcador de lesão tubular proximal. Pode ser detectada na biópsia renal e urina de pacientes com necrose tubular aguda isquêmica ${ }^{20}$.

A lesão renal é ainda pouco compreendida e uma das razões é a existência de marcadores pouco precisos e sem mensuração direta. Estudos sobre aspectos da disfunção renal freqüentemente utilizam a creatinina como sinalizador, entretanto a sua elevação sérica só ocorre quando já existe um comprometimento renal estabelecido.

A lesão renal é uma condição clínica comum durante a hospitalização principalmente em pacientes internados em UTI, ambiente no qual sua taxa de mortalidade aumenta independentemente de outros fatores ${ }^{11,21}$.

Nos últimos 20 anos, o progresso substancial na compreensão de mecanismos celulares e moleculares envolvendo a lesão renal aguda esclareceu mecanismos fisiopatológicos antes desconhecidos. Entretanto, esse conhecimento não tem se traduzido na aplicação de tratamentos efetivos e suficientemente específicos para prevenir ou excluir os agentes etiológicos. Essa limitação dificulta o controle de sua progressão. Tornando-se imperativa a necessidade de preencher essa lacuna de conhecimento no diagnóstico da lesão renal. 


\section{CONCLUSÕES}

Esse estudo demonstrou que nas últimas décadas houve um progresso no conhecimento sobre marcadores de função renal em decorrência da dificuldade, até os dias atuais, relacionados à prevenção e o tratamento da lesão renal aguda. Esse avanço contribuiu para a identificação de diversas proteínas, cuja finalidade é proporcionar a detecção precoce da disfunção renal e garantir ao paciente o diagnóstico preciso, que possibilite instituir tratamento adequado e individualizado, para o grau de comprometimento.

Entretanto, estudos multicêntricos que demonstrem maior abrangência, com amostras maiores são fundamentais para que se possam sugerir mudanças na rotina diagnóstica desta síndrome.

\section{REFERÊNCIAS}

01. Nolan CR, Anderson RJ - Hospital-acquired acute renal failure. J Am Soc Nephrol, 1998;9:710-718.

02. Frank R, Hargreaves R - Clinical biomarkers in drug discovery and development. Nat Rev Drug Discov, 2003;2:566-580.

03. De Gruttola VG, Clax P, DeMets DL et al - Considerations in the evaluation of surrogate endpoints in clinical trials: summary of a National Institutes of Health workshop. Control Clin Trials, 2001;22:485-502.

04. Han WK, Bonventre JV - Biologic markers for the early detection of acute kidney injury. Curr Opin Crit Care, 2004;10:476-482.

05. Herget-Rosenthal S, Marggraf G, Husing J et al - Early detection of acute renal failure by serum cystatin C. Kidney Int, 2004;66:1115-1122.

06. Parikh CR, Jani A, Melnikov VY et al - Urinary interleukin-18 is a marker of human acute tubular necrosis. Am J Kidney Dis, 2004;43:405-414.
07. Flynn FV - Assessment of renal function: selected developments. Clin Biochem, 1990;23:49-54.

08. Blaikley J, Sutton P, Walter M et al - Tubular proteinuria and enzymuria following open heart surgery. Intensive Care Med, 2003;29:1364-1367.

09. Mishra J, Mori K, Ma Q et al - Amelioration of ischemic acute renal injury by neutrophil gelatinase-associated lipocalin. J Am Soc Nephrol, 2004;15:3073-3082.

10. Hewitt SM, Dear J, Star RA - Discovery of protein biomarkers for renal diseases. J Am Soc Nephrol, 2004;15:1677-1689.

11. Lameire N, Van Biesen W, Vanholder R - Acute renal failure. Lancet, 2005;365:417-430.

12. Boldt J, Brenner T, Lehmann A et al - Influence of two different volume replacement regimens on renal function in elderly patients undergoing cardiac surgery: comparison of a new starch preparation with gelatin. Intensive Care Med, 2003;29:763-769.

13. Bernard AM, Vyskocil AA, Mahieu $P$ et al - Assessment of urinary retinol-binding protein as an index of proximal tubular injury. Clin Chem, 1987;33:775-779.

14. Sundberg AG, Appelkvist EL, Backman L et al - Urinary pi-class glutathione transferase as an indicator of tubular damage in the human kidney. Nephron, 1994;67:308-316.

15. Westhuyzen J, Endre ZH, Reece G et al - Measurement of tubular enzymuria facilitates early detection of acute renal impairment in the intensive care unit. Nephrol Dial Transplant, 2003;18:543-551.

16. Mennone A, Biemesderfer D, Negoianu D et al - Role of sodium/hydrogen exchanger isoform NHE3 in fluid secretion and absorption in mouse and rat cholangiocytes. Am J Physiol Gastrointest Liver Physiol, 2001;280: G247-G254

17. Melnikov VY, Faubel S, Siegmund B et al - Neutrophil-independent mechanisms of caspase-1-and IL-18-mediated ischemic acute tubular necrosis in mice. J Clin Invest, 2002;110:1083-1091.

18. Abrahamson M, Olafsson I, Palsdottir A et al - Structure and expression of the human cystatin C gene. Biochem J, 1990;268:287-294.

19. Tenstad O, Roald AB, Grubb A et al - Renal handling of radiolabelled human cystatin C in the rat. Scand J Clin Lab Invest, 1996;56:409-414.

20. Han WK, Bailly V, Abichandani R et al - Kidney Injury Molecule-1 (KIM1): a novel biomarker for human renal proximal tubule injury. Kidney Int, 2002;62:237-244.

21. Thakar CV, Worley S, Arrigain S et al - Influence of renal dysfunction on mortality after cardiac surgery: modifying effect of preoperative renal function. Kidney Int, 2005;67:1112-1119. 